International Mathematical Forum, 2, 2007, no. 7, 297 - 314

\title{
The Laplace's and Steepest Descents Methods Revisited
}

\author{
Chelo Ferreira \\ Departamento de Matemática Aplicada \\ Universidad de Zaragoza, 50013-Zaragoza, Spain \\ cferrei@unizar.es \\ José L. López and Pedro Pagola \\ Departamento de Matemática e Informática \\ Universidad Pública de Navarra, 31006-Pamplona, Spain \\ jl.lopez@unavarra.es, pedro.pagola@unavarra.es \\ Ester Pérez Sinusía \\ Departamento de Matemática Aplicada \\ UNED, 28040-Madrid, Spain \\ eperez@ind.uned.es
}

\begin{abstract}
We revise Laplace's and Steepest Descents methods of asymptotic expansions of integrals. The main difficulties in these methods are originated by a change of variables and an eventual deformation of the integration contour. We present a simplification of these methods that only requires an expansion of the integrand at the critical point(s). In this way, the calculation of the coefficients of the asymptotic expansion is simpler. The simplification in the case of several relevant critical points is even more significant and requires multi-point Taylor expansions. The new method that we present here unifies Laplace's and Steepest Descents methods in one unique formulation. Uniformity properties of the method are discussed. Asymptotic expansions of the Bernoulli and Jacobi polynomials and of a generalized confluent hypergeometric function are given as illustration.
\end{abstract}

Mathematics Subject Classification: 41A60, 41A58, 33C65

Keywords: Asymptotic expansions, Laplace's method, Steepest Descents, Multi-point Taylor expansions 


\section{Introduction}

The method of Steepest Descents is one of the most known and reputable classical methods for finding the asymptotic behaviour of integrals. It was introduced by Debye [2] in a paper concerning Bessel functions of large order. This method applies to contour integrals of the form:

$$
F(z) \equiv \int_{C} e^{z f(w)} g(w) d w, \quad z \rightarrow \infty
$$

where $C$ is a path in the complex plane, $z \in \mathbb{C}$ and the functions $f$ and $g$ are smooth enough or analytic wherever it is needed to perform the manipulations described below.

The critical points of the integrand are the points where $f^{\prime}(w)$ vanishes (saddle points of $f(w)$ if it is analytic). These points may give the main contributions to the asymptotic behaviour of the function $F(z)$ for large values of $z$. Other points that may give significant contributions are the endpoints of the path $C$, if these are finite. In fact, if $C=[a, b] \subset \mathbb{R}$ and $f(w)$ is real, one tries to determine if $\Re(z) f(w)$ is maximal at one of the critical points or at the endpoints of $C$. If a critical point, say $w_{0} \in C$, gives the main contribution $\left(e^{\Re(z) f(w)}\right.$ has its peak value at $\left.w_{0}\right)$, the asymptotic expansion of $F(z)$ is obtained from a suitable change of variable and local expansions of $f(w)$ and $g(w)$ at $w_{0}$. This method is the also very famous Laplace's method and we refer the reader to the modern book of Wong [[10], chap. 2. sec. 1] for a very complete description of it. A more classical reference is [1].

For $C \subset \mathbb{C}$ and complex $f(w)$, if one of the above mentioned critical points is not located on $C$, and one has verified that this point will give the main contributions, one tries to deform the path $C \rightarrow \Gamma$ such that the new path $\Gamma$ goes through that critical point (in fact saddle point of $f(w)$ ), say $w_{0}$. This is the Steepest Descents method. This path $\Gamma$ is defined by the equation $\Im\left(z f((w))=\Im\left(z f\left(w_{0}\right)\right)\right.$. The conditions under which the deformation $C \rightarrow \Gamma$ is possible depend on the original path $C$, on the region of analyticity of the functions $f(w)$ and $g(w)$ and on the phase of the complex parameter $z$. In fact, the path of steepest descent is a path where $e^{z f(w)}$ does not oscillate and concentrates at $w_{0}$, the concentration being greater for larger values of $|z|$. We explain in Figure 1 the situation that the new path $\Gamma$, a steepest descent path, runs through $w_{0}$. For a comprehensive description of this method we refer the reader once more to Wong's book [[10], chap. 2. sec. 4] or [1].

The first step of the Steepest Descents method consists in the deformation $C \rightarrow \Gamma$. As in the Laplace's method, the second step consists in a suitable change of variable and local expansions of $f(w)$ and $g(w)$ at $w_{0}$. As well as in Laplace's method, this change of variable depends on the multiplicity of $w_{0}$ as zero of $f^{\prime}(w)[[10]$, chap. 2. secs. 1, 4]. 


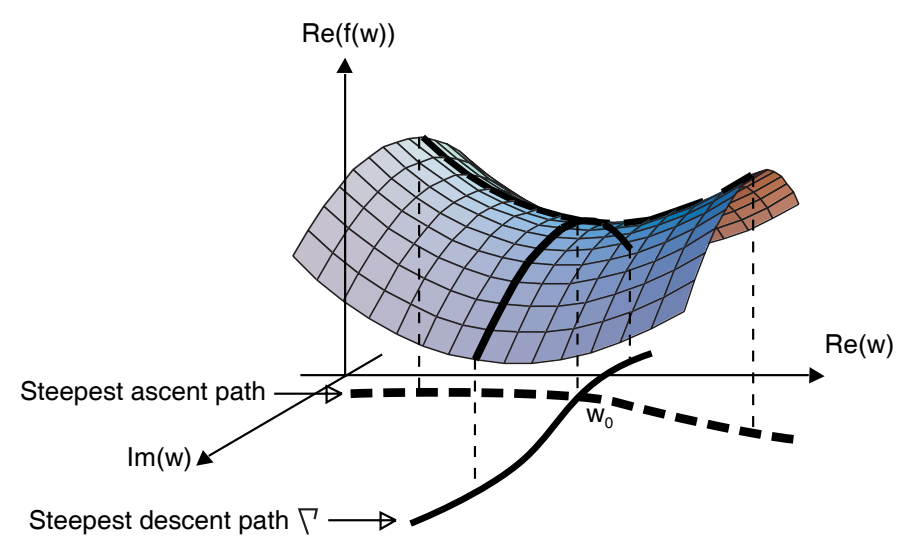

Figure 1: Typical plot of the real part of $f(w)$ over a simple saddle point $w_{0}$. Crossing this point we can find a steepest ascent path (dashed lines) and a steepest descent path $\Gamma$.

In the remaining of the paper, we will call asymptotic points to both, the critical points of $f(w)$ and the end points of $C$ (if a given point is both, a critical point and an end point, we call it critical point). If the function $f(w)$ has not one, but several asymptotic points: $\left\{w_{1}, w_{2}, \ldots\right\}$, then the dominant contribution to $F(z)$ comes from the most relevant asymptotic point. This is defined as the asymptotic point located on $C$ (or on $\Gamma$ if the deformation $C \rightarrow \Gamma$ is necessary) at which $\Re(z f(w))$ is maximal. Then the asymptotic expansion of $F(z)$ may be derived applying the Laplace's method (when $C=[a, b] \subset \mathbb{R}$ and $f(w)$ is real) or the Steepest Descents method (when $C \subset \mathbb{C}$ and $f(w)$ is complex) at that relevant asymptotic point. It may happens that this relevant asymptotic point is not unique. In this case, we must apply Laplace's method or Steepest Descents method at all of the relevant asymptotic points.

It is impossible to resume in few words the importance that these methods have had to derive asymptotic approximations of integrals in general, and of special functions in particular. In this way, it is very difficult to give an exhaustive list of the thousands of publications devoted to construct, generalize or apply to specific examples these methods. Consider that most of the known asymptotic expansions of special functions derived from integrals have been obtained by using one of these methods.

The main technical complication in the application of these methods lies in the identification of the steepest descents paths (in the Steepest Descents method) and in the change of variable (in both methods). Another source of complication is the existence of more than one relevant asymptotic point. Recently, some authors have suggested by means of specific examples that these methods may be simplified. The idea is just to expand $g(w)$ and perhaps also part of $e^{z f(w)}$ in (1) at the relevant asymptotic point(s) of $f(w)$ without 
any deformation of the integration path $C$ neither any change of variables. In this way, Paris has derived new and easier uniform asymptotic expansions of the incomplete gamma functions [8]. Temme and López have obtained quite easy uniform expansions of the Bernoulli, Euler, Charlier, Laguerre and Jacobi polynomials [4], [5]. But the proof of the asymptotic character of the expansions just mentioned is quite restricted to the examples on hand. In [8] that proof is based on a quite specific integral representation of the incomplete gamma functions, whereas in [4] and [5] the proofs are based on the "a priory" accidental convergence of the expansions for certain values of the uniformity parameter.

The purpose of this paper is to show that the success of that simplified version of the Laplace's or Steepest Descents methods used in those examples is not accidental and that works under mild conditions on $f(w)$ and $g(w)$. Then, we propose here a simplified version of the Laplace's or Steepest Descents methods with a simpler application that, in many situations, produces easier asymptotic expansions. We show that, if the Laplace or Steepest Descents methods apply to (1), then this simplified version applies too. It does not require any change of variable. Basically, it consists in an expansion of $g(w)$ at the relevant asymptotic point(s) of $f(w)$ and an interchange of sum and integral.

In the following section we introduce the method for the simplest case of having one unique asymptotic point. In section 3 we generalize the method to the case of two asymptotic points with the same multiplicity. In section 4 we consider an arbitrary number of asymptotic points with arbitrary multiplicities. Two examples are shown as illustration in section 5. In section 6 we discuss the uniform character of the method. In section 7 we write some final remarks and establish some conclusions.

\section{The simplest case: one asymptotic point}

For convenience, we rewrite (1) in the form

$$
F(z) \equiv \int_{a}^{b} e^{z f(w)} g(w) h(w) d w, \quad z \rightarrow \infty, \quad \Re z \geq z_{0}>0,
$$

where $a$ and $b$ are the end points of the path $C$ ( $a$ and $b$ may be finite or infinite and $a=b$ if $C$ is a closed loop). The functions $f(w), g(w)$ and $h(w)$ satisfy the hypotheses required by either, the Laplace's method or the Steepest Descents method. To be more precise:

i) $f(w), g(w)$ and $h(w)$ are analytic between $C$ and $\Gamma$ if a deformation of $C$ to a steepest descent path $\Gamma$ is required.

ii) The function $f(w)$ in (2) has only one asymptotic point $w_{0}: \Re[z(f(w)-$ $\left.\left.f\left(w_{0}\right)\right)\right]<0$ on $C$ and is bounded away from zero uniformly with respect to $z$ on 
$C$ except at $w=w_{0}$. If $C=[a, b] \subset \mathbb{R}, f(w)$ is real and $m$-times differentiable on $[a, b]$ and $w_{0} \in[a, b]$, then $w_{0}$ may be a critical point of multiplicity $m-1$ with $m$ even:

$$
f^{\prime}\left(w_{0}\right)=f^{\prime \prime}\left(w_{0}\right)=\ldots=f^{(m-1)}\left(w_{0}\right)=0, \quad f^{(m)}\left(w_{0}\right)<0,
$$

or an end point if $a \neq b: f^{\prime}\left(w_{0}\right)<0$ if $w_{0}=a$ or $f^{\prime}\left(w_{0}\right)>0$ if $w_{0}=b$ (in this case $m=1$ ). If $f(w)$ is complex (analytic between $C$ and $\Gamma$ ), then $w_{0}$ is a saddle point of $f$ of multiplicity $m-1$ :

$$
f^{\prime}\left(w_{0}\right)=f^{\prime \prime}\left(w_{0}\right)=\ldots=f^{(m-1)}\left(w_{0}\right)=0, \quad f^{(m)}\left(w_{0}\right) \neq 0 .
$$

iii) The function $g(w)$ has a Tayor expansion at $w_{0}$ :

$$
g(w)=\sum_{k=0}^{n-1} a_{k}\left(w-w_{0}\right)^{k}+g_{n}(w), \quad a_{k} \equiv \frac{g^{(k)}\left(w_{0}\right)}{k !}, \quad n=1,2,3, \ldots
$$

with $g_{n}(w)=\mathcal{O}\left(\left(w-w_{0}\right)^{n}\right)$ when $w \rightarrow w_{0}$.

iv) $h(w)$ is continuous on $C \backslash\left\{w_{0}\right\}$ and $h(w)=\mathcal{O}\left(\left(w-w_{0}\right)^{\alpha}\right)$ when $w \rightarrow w_{0}$ with $\Re \alpha>-1$.

v) The integral (2) and the integrals $\int_{a}^{b} e^{z f(w)} w^{k} h(w) d w, k=0,1,2, \ldots$ converge absolutely and uniformly with respect to $z$. The same holds for the integrals $\int_{\Gamma} e^{z f(w)} w^{k} h(w) d w$ if the deformation $C \rightarrow \Gamma$ is required.

If $f(w)$ is real and smooth enough on $C$, and $w_{0} \in C$, Laplace's method says that we must perform in (2) the change of variable $w \rightarrow u$ defined by $f(w)-f\left(w_{0}\right)=f^{(m)}\left(w_{0}\right) u$ and then approximate $w^{\prime}(u) g(w(u)) h(w(u))$ at $w=$ $w_{0}(u=0)$. If $w_{0} \notin C$ and $f(w)$ and $g(w) h(w)$ are analytic between $C$ and $\Gamma$, the Steepest Descents method says that we must deform the contour $C$ to the steepest descent path $\Gamma$ (which runs throw $w_{0}$ ) and then apply Laplace's method to the resulting integral over $\Gamma$. In any case, both methods tell us that the main contribution of the integrand in (2) to the value of the integral comes from the neighbourhood of the point $w_{0}$. We use this idea here in a more simple way: we approximate $g(w)$ at $w=w_{0}$ but without any change of variable neither a deformation of the contour $C$. We just introduce the expansion (3) in (2) and interchange sum and integral:

$$
\int_{a}^{b} e^{z f(w)} g(w) h(w) d w=\sum_{k=0}^{n-1} a_{k} \Phi_{k}(z)+R_{n}(z)
$$

with

$$
\Phi_{k}(z) \equiv \int_{a}^{b} e^{z f(w)} h(w)\left(w-w_{0}\right)^{k} d w
$$


and

$$
R_{n}(z) \equiv \int_{a}^{b} e^{z f(w)} h(w) g_{n}(w) d w
$$

These integrals exist thanks to the hypothesis v). Using iv) we see that

$$
\begin{aligned}
h(w)\left(w-w_{0}\right)^{k} & =\mathcal{O}\left(\left(w-w_{0}\right)^{k+\alpha}\right), & & w \rightarrow w_{0}, \\
h(w) g_{n}(w) & =\mathcal{O}\left(\left(w-w_{0}\right)^{n+\alpha}\right), & & w \rightarrow w_{0} .
\end{aligned}
$$

From hypothesis i), ii), iv) and v) and (7) we see that Laplace's or the Steepest Descents method can be applied to the integrals (5) and (6) to get their asymptotic behaviour for large $z$ [[10], chap. 2, secs. 1 or 4]:

$$
\Phi_{k}(z)=\mathcal{O}\left(\frac{e^{z f\left(w_{0}\right)}}{z^{(k+\alpha+1) / m}}\right), \quad R_{n}(z)=\mathcal{O}\left(\frac{e^{z f\left(w_{0}\right)}}{z^{(n+\alpha+1) / m}}\right), \quad z \rightarrow \infty .
$$

Therefore, (4)-(6) is an asymptotic expansion of $F(z)$ for large $z$.

\section{A first generalization: Two asymptotic points}

Suppose that $f(w)$ has two relevant asymptotic points. The philosophy contained in the above section tells us that we need to approach $g(w)$ at both asymptotic points. Then, condition ii) in that section is replaced by:

ii') The function $f(w)$ in (2) has two distinct asymptotic points $w_{1}$ and $w_{2}$ with $\Re\left(f\left(w_{1}\right)\right)<\Re\left(f\left(w_{2}\right)\right)\left(w_{2}\right.$ is dominant) or $\Re\left(f\left(w_{1}\right)\right)=\Re\left(f\left(w_{2}\right)\right)$ (both points are relevant). This means that $\Re\left[z\left(f(w)-f\left(w_{1}\right)\right)\right]<0$ on $C$ and is bounded away from zero uniformly with respect to $z$ on $C$ except at $w=w_{1}$ (and except at $w=w_{2}$ if $\left.\Re\left(f\left(w_{1}\right)\right)=\Re\left(f\left(w_{2}\right)\right)\right)$. For simplicity in the exposition and for its particular interest (see section 5 below) we consider in this section that both asymptotic points have the same multiplicity and postpone the general case to the next section. If $C=[a, b] \subset \mathbb{R}, f(w)$ is real and $m$-times differentiable on $[a, b]$ and $w_{1} \in[a, b]$ and $w_{2} \in[a, b]$, then $w_{1}$ and $w_{2}$ may be critical points of multiplicity $m-1$ with $m$ even:

$$
f^{\prime}\left(w_{s}\right)=f^{\prime \prime}\left(w_{s}\right)=\ldots=f^{(m-1)}\left(w_{s}\right)=0, \quad f^{(m)}\left(w_{s}\right)<0, \quad s=1,2,
$$

or end points if $a \neq b$ : $f^{\prime}\left(w_{1}\right)<0$ if $w_{1}=a$ or $f^{\prime}\left(w_{2}\right)>0$ if $w_{2}=b$ (in this case $m=1$ ). If $f(w)$ is complex (analytic between $C$ and $\Gamma$ ), then $w_{1}$ and $w_{2}$ are saddle points of $f$ of multiplicity $m-1$ :

$$
f^{\prime}\left(w_{s}\right)=f^{\prime \prime}\left(w_{s}\right)=\ldots=f^{(m-1)}\left(w_{s}\right)=0, \quad f^{(m)}\left(w_{s}\right) \neq 0, \quad s=1,2 .
$$

If $\Re\left(f\left(w_{1}\right)\right)=\Re\left(f\left(w_{2}\right)\right)$, the main contribution of the integrand in (2) to the value of the integral comes from the neighbourhood of both points $w_{1}$ and 
$w_{2}$. Then, we need to approximate $g(w)$ at both of them simultaneously and with the same accuracy. This means that we need the simultaneous Taylor expansion of $g(w)$ at $w_{1}$ and $w_{2}$. A detailed exposition of this may be found in [6]. We need to replace condition iii) and iv) in section 2 by:

iii') The function $g(w)$ has a two-point Taylor expansion at $w_{1}$ and $w_{2}$ :

$$
g(w)=\sum_{k=0}^{n-1}\left(a_{k}+b_{k} w\right)\left(w-w_{1}\right)^{k}\left(w-w_{2}\right)^{k}+g_{n}(w), \quad n=1,2,3, \ldots,
$$

with

$$
\begin{gathered}
a_{k} \equiv-w_{1} c_{k}\left(w_{1}, w_{2}\right)-w_{2} c_{k}\left(w_{2}, w_{1}\right), \quad b_{k} \equiv c_{k}\left(w_{1}, w_{2}\right)+c_{k}\left(w_{2}, w_{1}\right), \\
c_{0}\left(w_{1}, w_{2}\right) \equiv \frac{g\left(w_{2}\right)}{w_{2}-w_{1}}
\end{gathered}
$$

and, for $n=1,2,3, \ldots$,

$$
c_{n}\left(w_{1}, w_{2}\right) \equiv \sum_{k=0}^{n} \frac{(n+k-1) !}{k !(n-k) !} \frac{(-1)^{n+1} n g^{(n-k)}\left(w_{2}\right)+(-1)^{k} k g^{(n-k)}\left(w_{1}\right)}{n !\left(w_{1}-w_{2}\right)^{n+k+1}} .
$$

The remainder term verifies $g_{n}(w)=\mathcal{O}\left(\left(w-w_{1}\right)^{n}\right)$ when $w \rightarrow w_{1}$ and $g_{n}(w)=$ $\mathcal{O}\left(\left(w-w_{2}\right)^{n}\right)$ when $w \rightarrow w_{2}$.

iv') $h(w)$ is continuous on $C \backslash\left\{w_{1}, w_{2}\right\}$ and $h(w)=\mathcal{O}\left(\left(w-w_{1}\right)^{\alpha_{1}}\right)$ when $w \rightarrow w_{1}$ and $h(w)=\mathcal{O}\left(\left(w-w_{2}\right)^{\alpha_{2}}\right)$ when $w \rightarrow w_{2}$ with $\Re \alpha_{1}>-1$ and $\Re \alpha_{2}>-1$.

Introducing the expansion (9) in (2) and interchanging sum and integral we obtain

$$
\int_{a}^{b} e^{z f(w)} g(w) h(w) d w=\sum_{k=0}^{n-1}\left[a_{k} \Phi_{k}(z)+b_{k} \Psi_{k}(z)\right]+R_{n}(z)
$$

with

$$
\begin{aligned}
& \Phi_{k}(z) \equiv \int_{a}^{b} e^{z f(w)} h(w)\left(w-w_{1}\right)^{k}\left(w-w_{2}\right)^{k} d w \\
& \Psi_{k}(z) \equiv \int_{a}^{b} e^{z f(w)} h(w)\left(w-w_{1}\right)^{k}\left(w-w_{2}\right)^{k} w d w
\end{aligned}
$$

and

$$
R_{n}(z) \equiv \int_{a}^{b} e^{z f(w)} h(w) g_{n}(w) d w
$$


These integrals exist thanks to hypothesis v). Using iv') in these integrals we have that

$$
\begin{aligned}
h(w)\left(w-w_{1}\right)^{k}\left(w-w_{2}\right)^{k} & =\mathcal{O}\left(\left(w-w_{1}\right)^{k+\alpha_{1}}\right), & & w \rightarrow w_{1} \\
h(w)\left(w-w_{1}\right)^{k}\left(w-w_{2}\right)^{k} & =\mathcal{O}\left(\left(w-w_{2}\right)^{k+\alpha_{2}}\right), & & w \rightarrow w_{2} \\
h(w) g_{n}(w) & =\mathcal{O}\left(\left(w-w_{1}\right)^{n+\alpha_{1}}\right), & & w \rightarrow w_{1} \\
h(w) g_{n}(w) & =\mathcal{O}\left(\left(w-w_{2}\right)^{n+\alpha_{2}}\right), & & w \rightarrow w_{2} .
\end{aligned}
$$

From hypothesis i), ii'), iv') and v) and (16) we see that Laplace's method or the Steepest Descents method can be applied to the integrals (14) and (15) to get their asymptotic behaviour for large $z$ [[10], chap.2, secs. 1 and 4]:

$$
\Phi_{k}(z), \Psi_{k}(z)=\mathcal{O}\left(\frac{e^{z f\left(w_{2}\right)}}{z^{(k+\alpha+1) / m}}\right), \quad z \rightarrow \infty,
$$

where $\alpha \equiv \min \left\{\alpha_{1}, \alpha_{2}\right\}$. And

$$
R_{n}(z)=\mathcal{O}\left(\frac{e^{z f\left(w_{2}\right)}}{z^{(n+\alpha+1) / m}}\right), \quad z \rightarrow \infty .
$$

Therefore, (13)-(15) is an asymptotic expansion of $F(z)$ for large $z$.

If the multiplicities of $w_{1}$ and $w_{2}$ as zeros of $f^{\prime}(w)$ are different, the above approach is a little bit more cumbersome. We consider this situation in the following section, where we also consider $N$ relevant asymptotic points.

\section{The general case: several asymptotic points}

We consider in this section the most general case: $f(w)$ has $N$ asymptotic points of multiplicities $m_{1}-1, \ldots, m_{N}-1$. Condition ii) in section 2 must be replaced by:

ii" ) The function $f(w)$ in (2) has $N$ distinct asymptotic points $w_{1}, w_{2}, \ldots, w_{N}$ of multiplicities $m_{1}-1, m_{2}-1, \ldots, m_{N}-1$ respectively with $\Re\left(f\left(w_{1}\right)\right) \leq$ $\ldots \leq \Re\left(f\left(w_{N}\right)\right)$ (the number of relevant asymptotic points may be $1,2, \ldots$, or $N)$. This means that $\Re\left[z\left(f(w)-f\left(w_{1}\right)\right)\right]<0$ on $C$ and is bounded away from zero uniformly with respect to $z$ on $C$ except at $w=w_{1}$ (and perhaps at $\left.w_{2}, w_{3}, \ldots\right)$. If $C=[a, b] \subset \mathbb{R}, f(w)$ is real and $m$-times differentiable on $[a, b]$ and $w_{s} \in[a, b]$ for $s=1,2,3, \ldots, N$ and every $w_{s}$ may be a critical point of multiplicity $m_{s}-1$ with $m_{s}$ even:

$$
f^{\prime}\left(w_{s}\right)=f^{\prime \prime}\left(w_{s}\right)=\ldots=f^{\left(m_{s}-1\right)}\left(w_{s}\right)=0, \quad f^{\left(m_{s}\right)}\left(w_{s}\right)<0, \quad s=1,2, \ldots, N,
$$

or and end point if $a \neq b: f^{\prime}\left(w_{s}\right)<0$ if $w_{s}=a$ or $f^{\prime}\left(w_{s}\right)>0$ if $w_{s}=b$ for some $s$ (in this case $m_{s}=1$ ). If $f(w)$ is complex (analytic between $C$ and $\Gamma$ ), then $w_{s}$ are saddle points of multiplicity $m_{s}-1$ :

$$
f^{\prime}\left(w_{s}\right)=f^{\prime \prime}\left(w_{s}\right)=\ldots=f^{\left(m_{s}-1\right)}\left(w_{s}\right)=0, \quad f^{\left(m_{s}\right)}\left(w_{s}\right) \neq 0, \quad s=1,2,3, \ldots, N .
$$


The main contribution of the integrand in (2) to the value of the integral comes from the neighbourhood of all of the relevant asymptotic points. Then, we need to approximate $g(w)$ at all of them simultaneously. But as it will be clear below, the degree of accuracy in the approximation of $g(w)$ at $w_{s}$ must depend on the multiplicity of $w_{s}$ as zero of $f^{\prime}(w)$. This means that we need the multi-point Taylor expansion of $g(w)$ at the points $w_{1}, w_{2}, \ldots, w_{N}$ with $w_{1}$ repeated $m_{1}$ times, $w_{2}$ repeated $m_{2}$ times, .... A detailed exposition of this may be found in [7]. Then, conditions iii) and iv) in section 2 must be replaced by:

iii") The function $g(w)$ has a multi-point Taylor expansion at $w_{1}$ ( $m_{1}$ times), $w_{2}$ ( $m_{2}$ times $), \ldots, w_{N}\left(m_{N}\right.$ times $)$ :

$$
g(w)=\sum_{k=0}^{n-1} p_{k}(w) \prod_{s=1}^{N}\left(w-w_{s}\right)^{k m_{s}}+g_{n}(w), \quad n=1,2,3, \ldots
$$

where $p_{k}(w)$ are polynomials of degree $p \equiv m_{1}+m_{2}+\ldots+m_{N}-1$ :

$$
p_{k}(w)=a_{0}^{(k)}+a_{1}^{(k)} w+\ldots+a_{p}^{(k)} w^{p}
$$

and $g_{n}(w)=\mathcal{O}\left(\left(w-w_{s}\right)^{n m_{s}}\right)$ when $w \rightarrow w_{s}$. Coefficients $a_{l}^{(k)}$ may be calculated taking into account that the polynomial in the right hand side of (19) is the interpolation polynomial of $g(w)$ at $w_{1}, w_{2}, \ldots, w_{N}$ with data $g\left(w_{s}\right), g^{\prime}\left(w_{s}\right), \ldots$, $g^{\left(n m_{s}-1\right)}\left(w_{s}\right), s=1,2, \ldots, N$. Or else [7]:

$$
p_{k}(w) \equiv \sum_{j=1}^{N} \frac{\prod_{s=1, s \neq j}^{N}\left(w-w_{s}\right)^{m_{s}}}{\prod_{s=1, s \neq j}^{N}\left(w_{j}-w_{s}\right)^{m_{s}}} \sum_{l=0}^{m_{j}-1} a_{k, j, l}\left(w-w_{j}\right)^{l},
$$

with

$$
\begin{aligned}
a_{k, j, l}= & \left.\frac{1}{\left(k m_{j}+l\right) !} \frac{d^{k m_{j}+l}}{d w^{k m_{j}+l}}\left[\frac{g(w)}{\prod_{s=1, s \neq j}^{N}\left(w-w_{s}\right)^{k m_{s}}}\right]\right|_{w=w_{j}} \\
& +\left.\sum_{r=1, r \neq j}^{N} \frac{1}{\left(k m_{k}-1\right) !} \frac{d^{k m_{k}-1}}{d w^{k m_{j}+l}}\left[\frac{g(w)}{\left(w-w_{j}\right)^{l+1} \prod_{s=1, s \neq r}^{N}\left(w-w_{s}\right)^{k m_{s}}}\right]\right|_{w=w_{r}} .
\end{aligned}
$$

iv") $h(w)$ is continuous on $C \backslash\left\{w_{s} ; s=1,2, \ldots, N\right\}$ and $h(w)=\mathcal{O}\left(\left(w-w_{s}\right)^{\alpha_{s}}\right)$ when $w \rightarrow w_{s}$ with $\Re \alpha_{s}>-1$.

Introducing the expansion (19) in (2) and interchanging sum and integral we obtain

$$
\int_{a}^{b} e^{z f(w)} h(w) g(w) d w=\sum_{k=0}^{n-1} \sum_{l=1}^{p} a_{l}^{(k)} \Phi_{k}^{(l)}(z)+R_{n}(z)
$$


with

$$
\Phi_{k}^{(l)}(z) \equiv \int_{a}^{b} e^{z f(w)} w^{l} h(w) \prod_{s=1}^{N}\left(w-w_{s}\right)^{k m_{s}} d w, \quad l=0,1,2, \ldots, p
$$

and

$$
R_{n}(z) \equiv \int_{a}^{b} e^{z f(w)} h(w) g_{n}(w) d w
$$

These integrals exist thanks to the hypothesis v). Using iv") we see that

$$
\begin{aligned}
h(w)\left(w-w_{s}\right)^{k m_{s}} & =\mathcal{O}\left(\left(w-w_{s}\right)^{k m_{s}+\alpha_{s}}\right), & & w \rightarrow w_{s} \\
h(w) g_{n}(w) & =\mathcal{O}\left(\left(w-w_{s}\right)^{n m_{s}+\alpha_{s}}\right), & & w \rightarrow w_{s} .
\end{aligned}
$$

From hypothesis i), ii"), iv") and v) and (25) we see that Laplace's method or the Steepest Descents method can be applied to the integrals (23) and (24) to get their asymptotic behaviour for large $z$ [[10], chap. 2, secs. 1 and 4]:

$$
\Phi_{k}^{(l)}(z)=\mathcal{O}\left(\frac{e^{z f\left(w_{N}\right)}}{z^{k+\rho}}\right), \quad z \rightarrow \infty, \quad l=0,1,2, \ldots, p,
$$

with $\rho \equiv \min _{s}\left\{\left(\alpha_{s}+1\right) / m_{s}\right\}$. And

$$
R_{n}(z)=\mathcal{O}\left(\frac{e^{z f\left(w_{N}\right)}}{z^{n+\rho}}\right), \quad z \rightarrow \infty
$$

Therefore, (22)-(24) is an asymptotic expansion of $F(z)$ for large $z$.

\section{$5 \quad$ Examples}

The method described in sections 2, 3 and 4 is extraordinarily simple. But this simplicity does not mean that it is not a powerful method. We consider in this section two examples: Bernoulli [4] and Jacobi [5] polynomials which show the power of the method (and of course its simplicity). We do not improve very much here the asymptotic features of the expansions given in [4], [5] but we think that they are quite illustrative examples of the method. The proofs given here are easier and sorter than the proofs given in [4] and [5].

\subsection{Bernoulli polynomials}

Consider the following integral representation of the Bernoulli polynomials:

$$
B_{n}\left(z+\frac{1}{2}\right)=\frac{n !}{2 \pi i} \int_{C} \frac{w e^{w z}}{2 \sinh (w / 2)} \frac{d w}{w^{n+1}}
$$


where the contour $C$ encircles the origin in the counterclockwise direction and contains no poles of $w \sinh ^{-1}(w / 2)$. The shift $1 / 2$ in the variable $z$ is introduced in order to have reflection symmetry $z \rightarrow-z$ in the polynomials. We want to approximate $B_{n}(z)$ for large $z$ and $n$ and write $(28)$ in the form

$$
B_{n}\left(n z+\frac{1}{2}\right)=\frac{n !}{2 \pi i} \int_{C} e^{n f(w)} h(w) g(w) d w, \quad n \rightarrow \infty
$$

with $f(w)=w z-\log w, h(w)=1 / w$ and $g(w)=(w / 2) / \sinh (w / 2)$. The unique saddle point of $f(w)$ is $w_{0}=1 / z$ and has multiplicity 1 . Applying the method described in section 2 we obtain the asymptotic expansion (4)-(6) of $B_{n}(n z+1 / 2)$ for large $n$ (with $\alpha=0$ ). For simplicity we only write explicitly the first two terms of the expansion:

$$
\begin{aligned}
B_{n}\left(n z+\frac{1}{2}\right)= & \frac{n^{n} z^{n-1}}{2 \sinh (1 / 2 z)}\{1 \\
& \left.+\left[1+4\left(z-\frac{1}{2} \operatorname{coth}\left(\frac{1}{2 z}\right)\right) \operatorname{coth}\left(\frac{1}{2 z}\right)\right] \frac{1}{8 n z^{2}}+\mathcal{O}\left(\frac{1}{n^{2}}\right)\right\} .
\end{aligned}
$$

This is an asymptotic expansion for large $n$ for those values of $z$ for which the Steepest Descents method can be applied to the integral (29). The steepest ascent and descent paths of $f(w)$ are (see fig. 2(a)):

$$
\begin{cases}w=r e^{-i \operatorname{Arg}(z)}, & 0 \leq r<\infty, \quad(\text { steepest ascent path) } \\ w=\frac{(\theta+\operatorname{Arg}(z)) e^{i \theta}}{|z| \sin (\theta+\operatorname{Arg}(z))}, & \theta \in(-\pi-\operatorname{Arg}(z), \pi-\operatorname{Arg}(z)), \text { (steepest descent path). }\end{cases}
$$

The real part of $f(w)$ in $(29)$ is negative over a straight path joining the ends of the steepest descent path at the infinity (dashed line in fig. 2(a)). Therefore, the contour $C$ may be deformed into the steepest descent path $\Gamma$ whenever the poles $\pm 2 \pi i$ of $g(w)$ are not inside $\Gamma$. This happens for $|\operatorname{Arg}(z)|<2 \pi \Re z-\pi / 2$.

\subsection{Jacobi polynomials}

Consider the following integral representation of the Jacobi polynomials for $x \in(-1,1)$ :

$$
P_{n}^{(\alpha, \beta)}(x)=\frac{1}{2 \pi i} \frac{(-1)^{n}}{2^{n}} \int_{C} \frac{(1-w-x)^{\alpha}(1+w+x)^{\beta}}{(1-x)^{\alpha}(1+x)^{\beta}} e^{n f(x, w)} \frac{d w}{w},
$$

where the function $f(x, w)$ is defined by

$$
f(x, w) \equiv \log (1+w+x)+\log (1-w-x)-\log w
$$


and $C$ is a simple closed contour, in the positive sense, around $w=0$. The points $w=-x \pm 1$ are outside the contour, and $(1-w-x)^{\alpha} /(1-x)^{\alpha}$ and $(1+w+x)^{\beta} /(1+x)^{\beta}$ are to be taken as unity when $w=0$. We want to approximate $P_{n}^{(\alpha, \beta)}(x)$ for large $n$.

The function $f(x, w)$ has two conjugate equally important (relevant) saddle points of multiplicity 1 :

$$
w_{1}=i \sqrt{1-x^{2}}, \quad w_{2}=-i \sqrt{1-x^{2}}
$$

Applying the method described in section 3 with $g(w)=(1-w-x)^{\alpha}(1+w+x)^{\beta}$ and $h(w)=1 / w$, we obtain the asymptotic expansion (see [5] for details):

$$
P_{n}^{(\alpha, \beta)}(x)=\sum_{k=0}^{\infty}\left[a_{k} \Phi_{k}(x, n)+b_{k} \Psi_{k}(x, n)\right]
$$

where $a_{k}$ and $b_{k}$ are given in (10)-(12) and $\Phi_{k}(n)$ and $\Psi_{k}(n)$ are given in (14). For example,

$$
\Phi_{0}(x, n)=\frac{(2 n) !}{2^{2 n}(n !)^{2}} T_{n}(x), \quad \Psi_{0}(x, n)=\frac{(2 n-1) !}{2^{2 n-1} n !(n-1) !}\left(x^{2}-1\right) U_{n-1}(x),
$$

where $T_{n}(x)$ and $U_{n}(x)$ are Chebyshev polynomials. Then,

$$
\begin{aligned}
& P_{n}^{(\alpha, \beta)}(x)=\frac{(2 n) !}{2^{2 n}(n !)^{2}}\left\{\frac{\Re\left[\left(1-x+i \sqrt{1-x^{2}}\right)^{\alpha+1 / 2}\left(1+x-i \sqrt{1-x^{2}}\right)^{\beta+1 / 2}\right]}{(1-x)^{\alpha+1 / 2}(1+x)^{\beta+1 / 2}} T_{n}(x)\right. \\
& -\frac{\Im\left[\left(1-x+i \sqrt{1-x^{2}}\right)^{\alpha+1 / 2}\left(1+x-i \sqrt{1-x^{2}}\right)^{\beta+1 / 2}\right]}{(1-x)^{\alpha+1 / 2}(1+x)^{\beta+1 / 2}} \frac{2(n-1)}{2 n-1} \sqrt{1-x^{2}} U_{n-1}(x) \\
& \left.+\mathcal{O}\left(\frac{1}{n}\right)\right\} .
\end{aligned}
$$

The expansion (34) is an asymptotic expansion for large $n$ for those values of $x$ for which the Steepest Descents method can be applied to the integral (31). The steepest ascent and descent paths of $f(w)$ are given by the functions $r_{ \pm}(\theta)$ solution of the equations (see fig. 2(b)):

$r^{2} \sin (2 \theta)+2 x r \sin \theta=\left[1+r^{2} \sin ^{2} \theta-(x+r \cos \theta)^{2}\right] \tan \left[-\theta \pm \frac{\pi}{2} \pm \arctan \left(\frac{x}{\sqrt{1-x^{2}}}\right)\right]$.

The steepest descent path does not cross the branches $(-\infty,-x-1)$ and $(1-x, \infty)$ of $g(w)$. Therefore, the contour $C$ may be deformed into the steepest descent path $\Gamma$ for $-1<x<1$. 


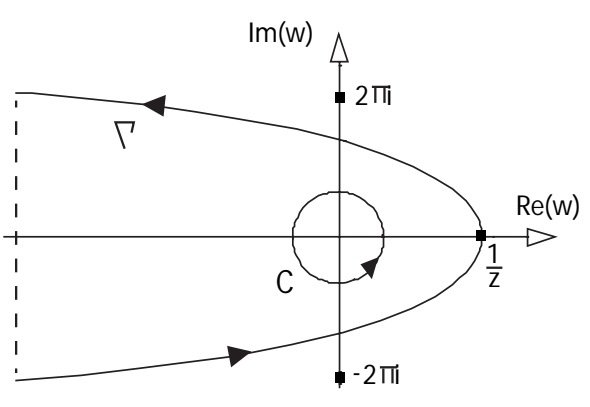

(a)

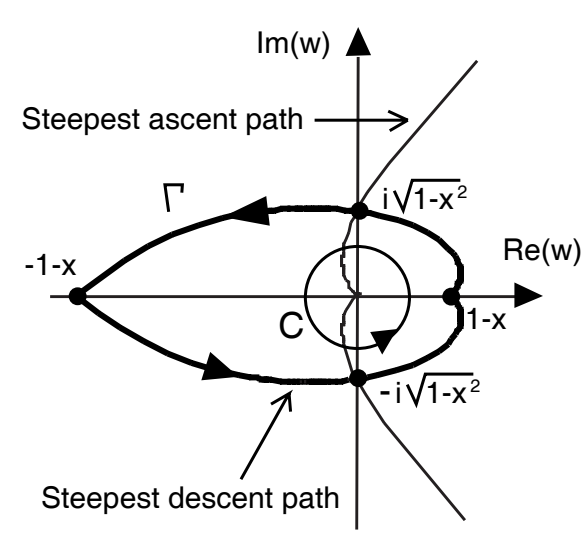

(b)

Figure 2: (a) Steepest descent path of the function $f(w)=z w-\log w$ for positive $z$. The condition $|\operatorname{Arg}(z)|<2 \pi \Re z-\pi / 2$ guarantees that the poles of the function $(w / 2) / \sinh (w / 2)$ lie outside the steepest descent path $\Gamma$ and the contour $C$ may be deformed into $\Gamma$. (b) Steepest descent and ascent paths of the function (32). The branch points of the function $g(w)=(1-w-x)^{\alpha}(1+w+x)^{\beta}$ lie outside the steepest descent path $\Gamma$ and the contour $C$ may be deformed into $\Gamma$.

\section{Uniformity aspects of the method}

If the function $f(w)$ in the integrand (2) contains a real parameter, say $a$, the saddle points of $f(w)=f(w, a)$ may depend on $a$. Then, it may happen that when $a$ crosses a critical value, an asymptotic point of $f(w)$ changes its character from non-relevant to relevant or vice-versa, or that two relevant asymptotic points coalesce, .... That is, the asymptotic behaviour of the integral may change drastically at certain values of $a$. It is well known that, in this case, the standard Laplace's or Steepest Descents methods must be modified to get asymptotic expansions of (2) uniformly valid for varying a [[10], chap. 7]. That modifications depend on the kind of coalescence of asymptotic points. We are speaking about the well known uniform asymptotic methods. For example, if two saddle points coalesce, then (2) may be approximated by Airy functions; if a saddle point coalesce with and end point, (2) may approximated by parabolic cylinder functions, .... .

The method described in sections $2-4$ does not need any modification because it is already a uniform method. Consider the following two possibilities (not mutually excluding) for the critical points of $f(w, a)$ when $a$ crosses a certain critical value $a^{*}$ :

a) Two or more relevant asymptotic points (critical or end points) coalesce. In this case the method described in section 4 is uniform. This is because the expansion of $g(w)$ is simultaneous at all of those asymptotic points 
and, if they coalesce, the formulation of the method doesn't change: if a critical point $w_{1}$ of multiplicity $m_{1}$ coalesce with another critical point $w_{2}$ of multiplicity $m_{2}$, then the factor $\left(w-w_{1}\right)^{n m_{1}}\left(w-w_{2}\right)^{n m_{2}}$ in the multi-Taylor expansion (19) becomes the factor $\left(w-w_{1}\right)^{n\left(m_{1}+m_{2}\right)}$. The only tricky point here is that if $w_{1} \rightarrow w_{2}$, terms of the form $0 / 0$ appear in the coefficients of the polynomial $p_{k}(w)$ defined in $(20)$. Then, this polynomial must be defined as the limit $w_{1} \rightarrow w_{2}$ of the right hand side of (20) [7].

b) One ore more asymptotic points $w_{s}(a)$ of $f(w, a)$ change their character from non-relevant to relevant or vice-versa when $a$ crosses a certain critical value $a^{*}$. In this case the method described in section 4 is obviously uniform. The only point here is that the corresponding exponential factors $e^{z f\left(w_{s}, a\right)}$ in the expansion of $F(z)$ change their character from relevant to non-relevant or viceversa when $a$ crosses $a^{*}$.

The following example shows the intrinsic uniform character of the method.

\subsection{A generalized confluent hypergeometric function}

We want to approximate the following integral:

$$
F(\alpha, b, c, x)=\int_{0}^{\infty} e^{-x t}(1+t)^{b}(c+t)^{\alpha} d t, \quad x>0, \quad c \geq 0, \quad \alpha, b \in \mathbb{R},
$$

for large $x$ and $b$. If $c=0$ then $\alpha>-1$. (For $c=0, F$ is a confluent hypergeometric function: $F(\alpha, b, 0, x)=U(\alpha+1, b+\alpha+1, x))$. We write

$$
F(\alpha, b, c, x)=\int_{0}^{\infty} e^{x f(t, a)} g(t) d t
$$

where $g(t)=(c+t)^{\alpha}$, the phase function is $f(t, a)=a \log (1+t)-t$ and $a \equiv b / x \in(-\infty, \infty)$ is the uniformity parameter. The unique critical point of this phase function is $t_{0}=a-1$ and has multiplicity 1 . It coalesces with the end point $t=0$ (which has multiplicity 0 ) when $a=1$. The character of these points (relevant or non-relevant) depend on the value of $a$ (see fig. 3). We need the three-point Taylor expansion of $g(t)$ at $t=0$ once and at $t=t_{0}$ twice. From formulas (19)-(21) with $w$ replaced by $t$ and $w_{1}=0, m_{1}=1$, $w_{2}=a-1$ and $m_{2}=2$ :

$$
g(t)=\sum_{k=0}^{n-1}\left(a_{0}^{(k)}+a_{1}^{(k)} t+a_{2}^{(k)} t^{2}\right) t^{k}(t-a+1)^{2 k}+g_{n}(t)
$$


Coefficients $a_{l}^{(k)}, l=0,1,2$, are regular when $t_{0} \rightarrow 0(a \rightarrow 1)$ if $c>0$ or if $c=0$ and $n \leq \alpha[6]$. For example, $a_{0}^{(0)}=c^{\alpha}$,

$$
a_{1}^{(0)}= \begin{cases}\frac{(c+a-1)^{\alpha-1}[2 c+\alpha+a(2-\alpha)-2]}{a-1} & \text { if } a \neq 1, \\ \alpha c^{\alpha-1} & \text { if } a=1,\end{cases}
$$

and

$$
a_{2}^{(0)}= \begin{cases}\frac{\alpha(a-1)(c+a-1)^{\alpha-1}+c^{\alpha}-(c+a-1)^{\alpha}}{(a-1)^{2}} & \text { if } a \neq 1, \\ \frac{\alpha(\alpha-1) c^{\alpha-2}}{2} & \text { if } a=1 .\end{cases}
$$

The functions $\Phi_{0}^{(0)}(x), \Phi_{0}^{(1)}(x)$ and $\Phi_{0}^{(2)}(x)$ in $(23)$ are

$$
\begin{gathered}
\Phi_{0}^{(0)}(x)=\int_{0}^{\infty} e^{-x t}(1+t)^{b} d t=\frac{e^{x}}{x^{b+1}} \Gamma(b+1, x), \\
\Phi_{0}^{(1)}(x)=\int_{0}^{\infty} e^{-x t}(1+t)^{b} t d t=\frac{e^{x}}{x^{b+1}}\left[\frac{\Gamma(b+2, x)}{x}-\Gamma(b+1, x)\right], \\
\Phi_{0}^{(2)}(x)=\int_{0}^{\infty} e^{-x t}(1+t)^{b} t^{2} d t=\frac{e^{x}}{x^{b+1}}\left[\frac{\Gamma(b+3, x)}{x^{2}}-2 \frac{\Gamma(b+2, x)}{x}+\Gamma(b+1, x)\right] .
\end{gathered}
$$

Therefore, for large $x$,

$$
\begin{aligned}
F(\alpha, b, c, x)= & \frac{e^{x}}{x^{b+1}} \Gamma(b+1, x)\left\{c^{\alpha}+a_{1}^{(0)}\left[\frac{\Gamma(b+2, x)}{x \Gamma(b+1, x)}-1\right]\right. \\
& \left.+a_{2}^{(0)}\left[\frac{\Gamma(b+3, x)}{x^{2} \Gamma(b+1, x)}-2 \frac{\Gamma(b+2, x)}{x \Gamma(b+1, x)}+1\right]+\mathcal{O}\left(\frac{1}{x}\right)\right\}
\end{aligned}
$$

uniformly for $a=b / x \in\left[a_{0}, a_{1}\right]$ with $a_{0}, a_{1} \in \mathbb{R}$ and $\alpha \geq 2$ if $c=0$.

\section{Concluding remarks}

The asymptotic expansion of the integral (2) for large $z$ is determined by the number, multiplicity and nature of the relevant asymptotic points of the exponent $f(w)$. Then, only the value of $h(w) g(w)$ around those points is of importance and a simultaneous approximation of $g(w)$ at those points is in order. But this approximation must have an appropriate contact order at each of those points according to their multiplicity. This is achieved by the multi-point Taylor expansion of $g(w)$ at those points counting multiplicities $[7]$.

The method described in this paper only invokes the classical Laplace's or Steepest Descents methods to show the asymptotic behaviour of the terms of the expansion and of the remainder. The asymptotic expansion is derived 


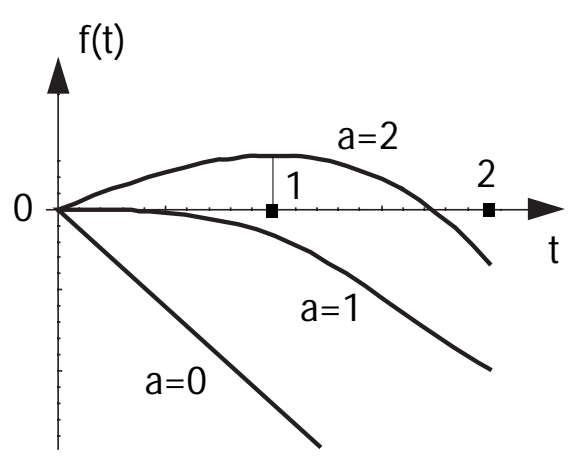

Figure 3: Plot of $f(t)=a \log (1+t)-t$ for $t \in[0,2]$ and three values of $a$. The relevant critical point (located at $t=a-1$ ) may be an interior point of the integration path, and end point or an exterior point depending on the uniformity parameter $a$. The critical point $t=a-1$ is the relevant asymptotic point for $a>1$, whereas the end point $t=0$ is the relevant asymptotic point for $a<1$. Both coalesce (and are relevant) if $a=1$.

quite easily by just introducing in (2) the multi-point Taylor expansion of $g(w)$ at the asymptotic points (19) and interchanging sum and integral to get (22).

We have split out a factor $h(w)$ from $g(w)$ in the integrand in (2). We did this in order to consider the following possibility: one factor of the integrand $(g(w))$ has a multi-point Taylor expansion at the asymptotic points of $f(w)$ and the other factor $(h(w))$ has not. Nevertheless, the integrability of the integral (2) requires a not too wild behaviour of $h(w)$ at those points (conditions iv), iv'), iv")).

The number of different asymptotic sequences involved in the expansions $\Phi_{k}^{(l)}(z)$ equals the number of asymptotic points of the exponent $f(w)$ in $(2)$. The multiplicities of those asymptotic points determine the asymptotic behaviour of the terms of the asymptotic sequences (the number $\rho$ in (26).

The expansions given in [4] and [5] are given in terms of an asymptotic sequence $\Phi_{k}(n)$ which verifies $\Phi_{k}(n)=\mathcal{O}\left(n^{\lfloor k / 2\rfloor-k}\right)$ as $n \rightarrow \infty$. The proof of this is based in a complicated recurrence for the $\Phi_{k}(n)$. Here we have seen that, in general, the asymptotic property of the $\Phi_{k}(n)$ is smoother: $\Phi_{k}(n)=$ $\mathcal{O}\left(n^{-k / 2}\right)$.

Standard Laplace's and Steepest Descents methods have been used to derive many (uniform and non-uniform) asymptotic expansions of special functions. Therefore, the alternative method described in this paper may be used to derive alternative asymptotic expansions of special functions. The examples given above show that, despite the apparent simplicity of the method, it is worth to consider this technique to generate new asymptotic expansions of special functions. 
In the standard Laplace's or Saddle point methods, the asymptotic expansion of the integral has a form similar to (4). The sequence $\Phi_{k}(z)$ in those methods is usually very simple: it is proportional to $z^{-k}$. But the coefficients $a_{k}$ are usually very complicated: they are the Taylor coefficients of a complicated function obtained after a change of variable (including a jacobian). On the other hand, the sequence $\Phi_{k}(z)$ in this method is more complicated (formula (5)) but the coefficients $a_{k}$ are simpler: they are the Taylor coefficients of the function $g(w)$ at $w=w_{0}$ (formula (3)). We can say that, with respect to the standard methods, in this method there is a movement of difficulty from the coefficients $a_{k}$ to the sequence $\Phi_{k}(z)$. The complexity of the sequence $\Phi_{k}(z)$ will depend of course on the precise example analyzed (in all the examples considered [3], [4], [5] and the example 6.1 the sequence $\Phi_{k}(z)$ turns out to be very simple).

If the integration path $C$ in the definition of $F(z)$ in $(2)$ contain relevant and non-relevant asymptotic points and uniformity is not a problem, we do not need to consider all of the asymptotic points simultaneously in the method. We just need to divide the integration path $C$ in several pieces containing less asymptotic points. Of course, the dominant asymptotic behaviour comes from the piece of $C$ containing the dominant asymptotic points. The contribution of the remaining pieces will be exponentially subdominant.

It has been pointed out in section 6 that this method is intrinsically uniform. Classical uniform methods based on standard Laplace's or Steepest Descents methods are strong modifications of the corresponding non-uniform methods. Moreover, there are several kind of modifications (and hence of uniform methods) depending on the kind of coalescence of asymptotic points (saddle point near an end point, two coalescing saddle points, ... ). This is so because every uniform version of the standard Laplace's or Steepest Descents methods requires an specific change of variable. But in the method described above we do not perform any change of variable, just expand $g(w)$ at the asymptotic points, whatever their dependence of the parameter $a$ described in section 6 is. Then, the method is intrinsically uniform, and moreover, it is unique independently of the kind of coalescence of asymptotic points.

Another source of asymptotic points (relevant for an asymptotic analysis) are the (possible) singularities of $h(w) g(w)$. We believe that in this case we just may introduce this new kind of asymptotic points in the list of asymptotic points and apply the above ideas. We think that in this case the multi-point Taylor expansion of $g(w)$ must be replaced by a multi-point Laurent or TaylorLaurent expansion [7]. This is subject of further investigation. 


\section{Acknowledgments}

We give thanks to Nico Temme for his helpful comments and improving suggestions. The Dirección General de Ciencia y Tecnología (REF. MTM2004-05221) is acknowledged by its financial support.

\section{References}

[1] N. Blestein and R. A. Handelsman, Asymptotic Expansions of Integrals, Dover Pub., New York, (1986).

[2] P. Debye, Näherungsformelm für die Zylinderfunktionene für grosse Werte des Arguments und unbeschränkt veränderliche Werte des Index, Math. Ann., 67 (1909), 535-558.

[3] C. Ferreira, J. L. Lopez and E. Perez Sinusia, The Gauss hypergeometric function $F(a, b ; c ; z)$ for large $b$ and $c$, accepted for publication in $J$. Comput. Appl. Math.

[4] J. L. Lopez and N. M. Temme, Uniform approximations of Bernoulli and Euler polynomials in terms of hyperbolic functions, Stud. Appl. Math., 103 (1999), 241-258.

[5] J. L. Lopez and N. M. Temme, Convergent asymptotic expansions of Charlier, Laguerre and Jacobi polynomials, Proc. Roy. Soc. Edimb. A., 134A (2004), 537-555.

[6] J. L. Lopez and N. M. Temme, Two-points Taylor expansions of analytic functions, Stud. Appl. Math., 109 (2002), 297-311.

[7] J. L. Lopez and N. M. Temme, Multi-point Taylor expansions of analytic functions, Trans. Amer. Math. Soc., 356 (2004) 4323-4342.

[8] R. B. PAris, A uniform asymptotic expansion for the incomplete gamma function. J. Comput. Appl. Math., 148 (2002), 323-339.

[9] N.M. Temme, Special functions: An introduction to the classical functions of mathematical physics, Wiley and Sons, New York, (1996).

[10] R. Wong, Asymptotic Approximations of Integrals, Academic Press, New York, (1989).

\section{Received: April 27, 2006}

